\title{
Spatial Memory and Taxis-Driven Pattern Formation in Model Ecosystems
}

\author{
Jonathan R. Potts ${ }^{1}$ (D) Mark A. Lewis ${ }^{2,3}$
}

Received: 12 March 2019 / Accepted: 27 May 2019 / Published online: 4 June 2019

(c) The Author(s) 2019

\begin{abstract}
Mathematical models of spatial population dynamics typically focus on the interplay between dispersal events and birth/death processes. However, for many animal communities, significant arrangement in space can occur on shorter timescales, where births and deaths are negligible. This phenomenon is particularly prevalent in populations of larger, vertebrate animals who often reproduce only once per year or less. To understand spatial arrangements of animal communities on such timescales, we use a class of diffusion-taxis equations for modelling inter-population movement responses between $N \geq 2$ populations. These systems of equations incorporate the effect on animal movement of both the current presence of other populations and the memory of past presence encoded either in the environment or in the minds of animals. We give general criteria for the spontaneous formation of both stationary and oscillatory patterns, via linear pattern formation analysis. For $N=2$, we classify completely the pattern formation properties using a combination of linear analysis and nonlinear energy functionals. In this case, the only patterns that can occur asymptotically in time are stationary. However, for $N \geq 3$, oscillatory patterns can occur asymptotically, giving rise to a sequence of period-doubling bifurcations leading to patterns with no obvious regularity, a hallmark of chaos. Our study highlights the importance of understanding between-population animal movement for understanding spatial species distributions, something that is typically ignored in species distribution modelling, and so develops a new paradigm for spatial population dynamics.
\end{abstract}

Electronic supplementary material The online version of this article (https://doi.org/10.1007/s11538019-00626-9) contains supplementary material, which is available to authorized users.

$凶$ Jonathan R. Potts

j.potts@sheffield.ac.uk

1 School of Mathematics and Statistics, University of Sheffield, Hicks Building, Hounsfield Road, Sheffield S3 7RH, UK

2 Department of Mathematical and Statistical Sciences, CAB632, University of Alberta, Edmonton, AB T6G 2G1, Canada

3 Department of Biological Sciences, CAB632, University of Alberta, Edmonton, AB T6G 2G1, Canada 
Keywords Advection-diffusion - Animal movement · Chaos · Movement ecology · Population dynamics · Taxis

\section{Introduction}

Mathematical modelling of spatial population dynamics has a long history of uncovering the mechanisms behind a variety of observed patterns, from predator-prey interactions (Pascual 1993; Lugo and McKane 2008; Sun et al. 2012) to biological invasions (Petrovskii et al. 2002; Hastings et al. 2005; Lewis et al. 2016) to interspecies competition (Hastings 1980; Durrett and Levin 1994; Girardin and Nadin 2015). These models typically start with a mathematical description of the birth and death processes and then add spatial aspects in the form of dispersal movements. Such movements are often assumed to be diffusive (Okubo and Levin 2013), but sometimes incorporate elements of taxis (Kareiva and Odell 1987; Lee et al. 2009; Potts and Petrovskii 2017). The resulting models are often systems of reaction-advection-diffusion (RAD) equations, which are amenable to pattern formation analysis via a number of established mathematical techniques (Murray 2003).

An implicit assumption behind these RAD approaches is that the movement processes (advection and diffusion) take place on the same temporal scale as the birth and death processes (reaction). However, many organisms will undergo significant movement over much shorter timescales. For example, many larger animals (e.g. most birds, mammals, and reptiles) will reproduce only once per year, but may rearrange themselves in space quite considerably in the intervening period between natal events. These rearrangements can give rise to emergent phenomena such as the 'landscape of fear' (Laundré et al. 2010), aggregations of coexistent species (Murrell and Law 2003), territoriality (Potts and Lewis 2014), home ranges (Briscoe et al. 2002; Börger et al. 2008), and spatial segregation of interacting species (Shigesada et al. 1979).

Indeed, the study of organism movements has led, in the past decade or two, to the emergence of a whole subfield of ecology, dubbed 'movement ecology' (Nathan et al. 2008; Nathan and Giuggioli 2013). This is gaining increasing attention by both statisticians (Hooten et al. 2017) and empirical ecologists (Kays et al. 2015; Hays et al. 2016), in part driven by recent rapid technological advances in biologging (Williams et al., in review). Often, a stated reason for studying movement is to gain insight into space-use patterns (Vanak et al. 2013; Avgar et al. 2015; Fleming et al. 2015; Avgar et al. 2016). Yet despite this, we lack a good understanding of the spatial pattern formation properties of animal movement models over timescales where birth and death effects are minimal.

To help rectify this situation, we introduce here a class of models that focuses on one particular type of movement: taxis of a population in response to the current or recent presence of foreign populations. This covers several ideas within the ecological literature. One is the movement of a species away from areas where predator or competitor species reside, often dubbed the 'landscape of fear' (Laundré et al. 2010; Gallagher et al. 2017). The opposing phenomenon is that of predators moving towards prey, encapsulated in prey-taxis models (Kareiva and Odell 1987; Lee et al. 2009). Many species exhibit mutual avoidance, which can be either inter-species avoidance or 
intra-species avoidance. The latter gives rise to territoriality, and there is an established history of modelling efforts devoted to its study (Adams 2001; Lewis and Moorcroft 2006; Potts and Lewis 2014). Likewise, some species exhibit mutual attraction due to benefits of coexistence (Murrell and Law 2003; Kneitel and Chase 2004; Vanak et al. 2013). Since some of these phenomena are inter-specific and others are intra-specific, we use the word 'population' to mean a group of organisms that are all modelled using the same equation, noting once and for all that 'population' may be used to mean an entire species (for modelling inter-species interactions, e.g. the landscape of fear), or it may refer to a group within a single species (for intra-species interactions, e.g. territoriality).

There are various processes by which one population can sense the presence of others. One is by directly sensing organism presence by sight or touch. However, it is perhaps more common for the presence of others to be advertised indirectly. This could either be due to marks left in the landscape, a process sometimes known as stigmergy (Giuggioli et al. 2013), or due to memory of past interactions (Fagan et al. 2013; Potts and Lewis 2016a). We show here that these three interaction processes (direct, stigmergic, memory) can all be subsumed under a single modelling framework.

The resulting model is a system of $N$ diffusion-taxis equations, one for each of $N$ populations. We analyse this system using a combination of linear pattern formation analysis (Turing 1952), energy functionals (nonlinear), and numerical bifurcation analysis. We classify completely the pattern formation properties for $N=2$, noting that here only stationary patterns can form. For $N=3$, we show that, as well as there being parameter regimes where stationary patterns emerge, oscillatory patterns can emerge for certain parameter values, where patterns remain transient and never settle to a steady state. In these regimes, we observe both periodic behaviour and behaviour where the period is much less regular. These irregular regimes emerge through a sequence of period-doubling bifurcations, a phenomenon often associated with the emergence of chaos.

The fact that inter-population taxis processes can give rise to perpetually changing, possibly chaotic, spatial patterns is a key insight into the study of species distributions. Researchers often look to explain such transient spatial patterns by examining changes in the underlying environment. However, we show that continually changing patterns can emerge without the need to impose any environmental effect. As such, our study highlights the importance of understanding inter-population movement responses for gaining a full understanding of the spatial distribution of ecological communities, and helps link movement ecology to population dynamics in a non-speculative way.

\section{The Modelling Framework}

Our general modelling framework considers $N$ populations, each of which has a fixed overall size. For each population, the constituent individuals move in space through a combination of a diffusive process and a tendency to move towards more attractive areas and away from those that are less attractive. Denoting by $u_{i}(\mathbf{x}, t)$ the probability density function of population $i$ at time $t(i \in\{1, \ldots, N\})$, and by $A_{i}(\mathbf{x}, t)$ the attrac- 
tiveness of location $\mathbf{x}$ to members of population $i$ at time $t$, we construct the following movement model

$$
\frac{\partial u_{i}}{\partial t}=D_{i} \nabla^{2} u_{i}-c_{i} \nabla \cdot\left(u_{i} \nabla A_{i}\right),
$$

where $D_{i}>0$ is the magnitude of the diffusive movement of population $i$ and $c_{i} \geq 0$ is the magnitude of the drift tendency towards more attractive parts of the landscape.

Here, we assume that the attractiveness of a point $\mathbf{x}$ on the landscape at time $t$ is determined by the presence of individuals from other populations. We look at three scenarios. For some organisms, particularly very small ones such as amoeba, there may be sufficiently many individuals constituting each population so that the probability density function is an accurate descriptor of the number of individuals present at each part of space. This is Scenario 1. In this case, the attractiveness of a part of space to population $i$ may simply be proportional to the weighted sum of the probability density functions of all the other populations, or possibly a locally averaged probability density. In other words

$$
\text { Scenario 1: } A_{i}(\mathbf{x}, t)=\sum_{j \neq i} a_{i j} \bar{u}_{j}(\mathbf{x}, t),
$$

where $a_{i j}$ are constants, which can be either positive, if population $i$ benefits from the presence of population $j$, or negative, if population $i$ seeks to avoid population $j$, and

$$
\bar{u}_{j}(\mathbf{x}, t)=\frac{1}{\left|C_{\mathbf{x}}\right|} \int_{C_{\mathbf{x}}} u_{j}(\mathbf{z}, t) \mathrm{d} \mathbf{z},
$$

where $C_{\mathbf{x}}$ is a small neighbourhood of $\mathbf{x}$ and $\left|C_{\mathbf{x}}\right|$ is the Lebesgue measure of $C_{\mathbf{x}}$. The importance of this spatial averaging will become apparent in Sect. 3.

For larger organisms (e.g. mammals, birds, reptiles, etc.), individuals may be more spread out on the landscape. Here, the presence may be advertised by one of two processes (Scenarios 2 and 3). In Scenario 2, we model individuals as leaving marks on the landscape (e.g. urine, faeces, footprints etc.) to which individuals of the other populations respond. Denoting by $p_{i}$ the presence of marks that are foreign to population $i$, we can model this using the following differential equation (cf. Lewis and Murray 1993; Lewis and Moorcroft 2006; Potts and Lewis 2016b)

$$
\frac{\partial p_{i}}{\partial t}=\sum_{j \neq i} \alpha_{i j} u_{j}-\mu p_{i}
$$

where $\mu>0$ and $\alpha_{i j} \in \mathbb{R}$ are constants. If $\alpha_{i j}>0$ (resp. $\alpha_{i j}<0$ ), then population $i$ is attracted towards (resp. repelled away from), population $j$. In this scenario, we model $A_{i}(\mathbf{x}, t)$ as a spatial averaging of $p_{i}(\mathbf{x}, t)$ so that

$$
\text { Scenario 2: } A_{i}(\mathbf{x}, t)=\bar{p}_{i}(\mathbf{x}, t) \text {, }
$$

where $\bar{p}_{i}(\mathbf{x}, t)$ is defined in an analogous way to $\bar{u}_{j}(\mathbf{x}, t)$ in Eq. (3). 
Finally, Scenario 3 involves individuals remembering places where they have had recent encounters with individuals of another population, and moving in a manner consistent with a cognitive map. We assume here that individuals within a population are able to transmit information between themselves so that they all share common information regarding the expected presence of other populations, which we denote by $k_{i}(\mathbf{x}, t)$ for population $i$. This can be modelled as follows (cf. Potts and Lewis 2016a)

$$
\frac{\partial k_{i}}{\partial t}=\sum_{j \neq i} \beta_{i j} u_{i} u_{j}-\left(\zeta+v u_{i}\right) k_{i}
$$

where $v>0, \zeta \geq 0$, and $\beta_{i j} \in \mathbb{R}$ are constants. Here, $\beta_{i j}$ refers to the tendency for animals from population $i$ to remember a spatial location, given an interaction with an individual from population $j, \zeta$ is the rate of memory decay, and $v$ refers to the tendency for animals from population $i$ to consider a location not part of $j$ 's range if individuals from $i$ visit that location without observing an individual from $j$ there. See Potts and Lewis (2016a) more explanation of the motivation and justification for the functional form in Eq. (6), in the context of avoidance mechanisms.

In this scenario, we model $A_{i}(\mathbf{x}, t)$ as a spatial averaging of $k_{i}(\mathbf{x}, t)$ so that

$$
\text { Scenario 3: } A_{i}(\mathbf{x}, t)=\bar{k}_{i}(\mathbf{x}, t) \text {, }
$$

where $\bar{k}_{i}(\mathbf{x}, t)$ is defined in an analogous way to $\bar{u}_{j}(\mathbf{x}, t)$ in Eq. (3).

Note the similarity between Scenarios 2 and 3 and the idea of a "landscape of fear", which has become increasingly popular in the empirical literature (Laundré et al. 2010). The landscape of fear invokes the idea that there are certain parts of space that individuals in a population tend to avoid because they perceive those areas to have a higher risk of aggressive interactions (either due to predation or competition). The degree to which this danger is perceived across space creates a spatial distribution of fear, and animals may be modelled as advecting down the gradient of this distribution.

\section{General Results in 1D}

Although our modelling framework can be defined in arbitrary dimensions, we will focus our analysis on the following 1D version of Eq. (1)

$$
\frac{\partial u_{i}}{\partial t}=D_{i} \frac{\partial^{2} u_{i}}{\partial x^{2}}-c_{i} \frac{\partial}{\partial x}\left(u_{i} \frac{\partial A_{i}}{\partial x}\right) .
$$

We also work on a line segment, so that $x \in[0, L]$ for some $L>0$.

It is convenient for analysis to assume that, for Scenarios 2 and 3, the quantities $p_{i}(x, t)$ and $k_{i}(x, t)$ equilibrate much faster than $u_{i}(x, t)$, so we can make the approximations $\partial p_{i} / \partial t \approx 0$ and $\partial k_{i} / \partial t \approx 0$. Making the further assumption that there is no memory decay ( $\zeta=0$ in Eq. 6 ), which turns out later to be convenient for unifying the three scenarios, we have the following approximate versions of Eqs. (5) and (7) 


$$
\begin{aligned}
& \text { Scenario 2: } A_{i}(x, t) \approx \sum_{j \neq i} \frac{\alpha_{i j}}{\mu} \bar{u}_{j}(x, t), \\
& \text { Scenario 3: } A_{i}(x, t) \approx \sum_{j \neq i} \frac{\beta_{i j}}{v} \bar{u}_{j}(x, t) .
\end{aligned}
$$

We non-dimensionalise our system by setting $\tilde{u}_{i}=L u_{i}, \tilde{x}=x / L, \tilde{t}=t D_{1} / L^{2}$, $d_{i}=D_{i} / D_{1}$ and

$$
\gamma_{i j}= \begin{cases}\frac{c_{i} a_{i j}}{L D_{1}}, & \text { in Scenario 1, } \\ \frac{c_{i} \alpha_{i j}}{\mu L D_{1}}, & \text { in Scenario 2, } \\ \frac{c_{i} \beta_{i j}}{\nu L D_{1}}, & \text { in Scenario 3. }\end{cases}
$$

Then, dropping the tildes over $\tilde{u}_{i}, \tilde{x}$, and $\tilde{t}$ for notational convenience, we obtain the following non-dimensional model for space use

$$
\frac{\partial u_{i}}{\partial t}=d_{i} \frac{\partial^{2} u_{i}}{\partial x^{2}}-\frac{\partial}{\partial x}\left(u_{i} \sum_{j \neq i} \gamma_{i j} \frac{\partial \bar{u}_{j}}{\partial x}\right)
$$

where $d_{1}=1$, by definition.

For simplicity, we assume that boundary conditions are periodic, so that

$$
u_{i}(0, t)=u_{i}(1, t)
$$

With this identification in place, we can define the 1D spatial averaging kernel from Eq. (3) to be $C_{x}=\{z \in[0,1] \mid(x-\delta)(\bmod 1)<z<(x+\delta)(\bmod 1)\}$ for $0<\delta \ll 1$. Here, $z(\bmod 1)$ is used so as to account for the periodic boundary conditions and is defined to be the unique real number $z^{\prime} \in[0,1)$ such that $z-z^{\prime} \in \mathbb{Z}$. Then, Eq. (3) becomes

$$
\bar{u}_{j}=\frac{1}{2 \delta} \int_{(x-\delta)(\bmod 1)}^{(x+\delta)(\bmod 1)} u_{j}(z, t) \mathrm{d} z .
$$

Finally, since $u_{i}(x, t)$ are probability density functions of $x$, defined on the interval $x \in[0,1]$, we also have the integral condition

$$
\int_{0}^{1} u_{i}(x, t) \mathrm{d} x=1
$$

This condition means that we have a unique spatially homogeneous steady state, given by $u_{i}^{*}(x)=1$ for all $i \in\{1, \ldots, N\}, x \in[0,1]$. Our first task for analysis is to see whether this steady state is unstable to non-constant perturbations. 
We set $\mathbf{w}(x, t)=\left(u_{1}-1, \ldots, u_{N}-1\right)^{\mathrm{T}}=\left(u_{1}^{(0)}, \ldots, u_{N}^{(0)}\right)^{\mathrm{T}} \exp (\sigma t+\mathrm{i} \kappa x)$, where $u_{1}^{(0)}, \ldots, u_{N}^{(0)}$ and $\sigma, \kappa$ are constants, and the superscript $\mathrm{T}$ denotes matrix transpose. By neglecting nonlinear terms, Eq. (12) becomes

$$
\sigma \mathbf{w}=\kappa^{2} M(\kappa, \delta) \mathbf{w}
$$

where $M(\kappa, \delta)=\left[M_{i j}(\kappa, \delta)\right]_{i, j}$ is a matrix with

$$
M_{i j}(\kappa, \delta)= \begin{cases}-d_{i}, & \text { if } i=j, \\ \gamma_{i j} \operatorname{sinc}(\kappa \delta), & \text { otherwise }\end{cases}
$$

where $\operatorname{sinc}(\xi)=\sin (\xi) / \xi$. Therefore, patterns form whenever there is some $\kappa$ such that there is an eigenvalue of $M(\kappa, \delta)$ with positive real part.

It is instructive to examine the limit case $\delta \rightarrow 0$. Here

$$
M_{i j}(\kappa, 0)=\left\{\begin{array}{lc}
-d_{i}, & \text { if } i=j \\
\gamma_{i j}, & \text { otherwise. }
\end{array}\right.
$$

so $M_{i j}(\kappa, 0)$ is, in fact, independent of $\kappa$, and so we define the constant matrix $M_{0}=$ $\left[M_{i j}(\kappa, 0)\right]_{i, j}$. When $\delta \rightarrow 0$, there are two cases pertinent to pattern formation:

1. All the eigenvalues of $M_{0}$ have negative real part, in which case no patterns form.

2. At least one eigenvalue of $M_{0}$ has positive real part, in which case the dominant eigenvalue of $\kappa^{2} M_{0}$ is an increasing function of $\kappa$. Therefore, patterns can form at arbitrarily high wavenumbers. In other words, the pattern formation problem is ill-posed.

The problem posed by point (2) above can often be circumvented by using a strictly positive $\delta$. For example, Fig. 1 shows the dispersion relation (plotting the dominant eigenvalue against $\kappa$ ) for a simple case where $N=2, d_{i}=1, \gamma_{i j}=-5$ for all $i, j$, and $\delta$ is varied. In this example, the dominant eigenvalue is real for all $\kappa$. We see that, for $\delta \rightarrow 0$, the dispersion relation is monotonically increasing. However, a strictly positive $\delta$ means the eigenvalues are $\kappa^{2}[-2 \pm 5 \operatorname{sinc}(\kappa \delta)] / 2$, which is asymptotically $\sigma \approx-\kappa^{2}$ as $\kappa \rightarrow \infty$. Hence, the dominant eigenvalue is positive only for a finite range of $\kappa$ values, as long as $\delta>0$.

The fact that the pattern formation problem is ill-posed for $\delta \rightarrow 0$ suggests that classical solutions may not exist in this case. This phenomenon is not new and has been observed in very similar systems studied by Briscoe et al. (2002), Potts and Lewis (2016a,b). More generally, there are various studies that deal with regularisation of such ill-posed problems in slightly different contexts using other techniques, which incorporate existence proofs (e.g. Padrón 1998, 2004). We therefore conjecture that classical solutions do exist for the system given by Eq. (12) in the case where $\delta>0$, and the numerics detailed in this paper give evidence to support this. However, we do not prove this conjecture here, since it is a highly non-trivial question in general, and the purpose of this paper is just to introduce the model structure and investigate possible types of patterns that could arise. Nonetheless, it is an important subject for 

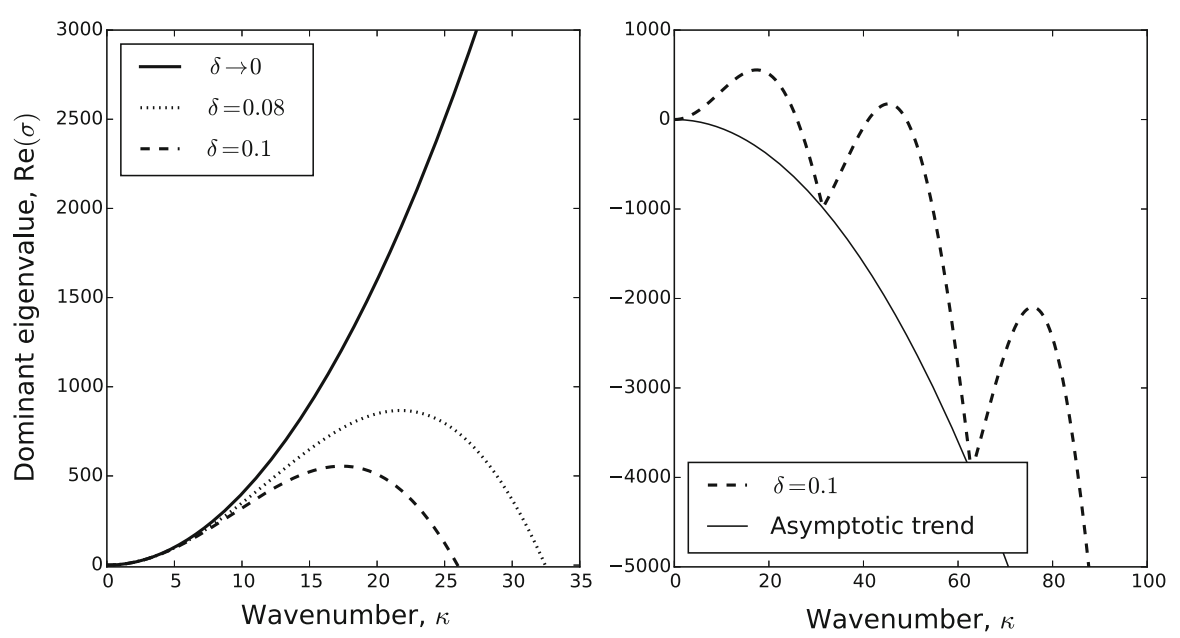

Fig. 1 Example dispersion relations. Here we give dispersion relations for the system described by Eq. (12) with $N=2, d_{i}=1$, and $\gamma_{i j}=-5$ for all $i, j$. In the left-hand panel, we examine three values of $\delta$, showing that, for $\delta \rightarrow 0$, the dispersion relation is monotonic, but this monotonicity is tamed by setting $\delta>0$. In the right-hand panel, we extend the dispersion relation plot for $\delta=0.1$ to a larger range of $\kappa$ values, together with the analytically derived asymptotic trend

future research. In the next two sections, we will examine specific cases where $N=2$ and $N=3$.

\section{The Case of Two Interacting Populations $(N=2)$}

When $N=2$, the system given by Eqs. $(12,14,15)$ is simple enough to categorise its linear pattern formation properties in full. Here

$$
M(\kappa, \delta)=\left(\begin{array}{cc}
-1 & \gamma_{12} \operatorname{sinc}(\kappa \delta) \\
\gamma_{21} \operatorname{sinc}(\kappa \delta) & -d_{2}
\end{array}\right)
$$

The eigenvalues of $M(\kappa, \delta)$ are therefore

$$
\sigma(\kappa)=\frac{-\left(1+d_{2}\right) \pm \sqrt{\left(1+d_{2}\right)^{2}+4\left[\gamma_{12} \gamma_{21} \operatorname{sinc}^{2}(\kappa \delta)-d_{2}\right]}}{2} .
$$

Notice first that if $\sigma(\kappa)$ is not real, then the real part is $\operatorname{Re}[\sigma(\kappa)]=-\left(1+d_{2}\right) / 2$, which is always negative, since $d_{2}>0$. Hence, patterns can only form when $\sigma(\kappa) \in \mathbb{R}$, meaning that the discriminant, $\Delta=\left(1+d_{2}\right)^{2}+4\left[\gamma_{12} \gamma_{21} \operatorname{sinc}^{2}(\kappa \delta)-d_{2}\right]$, must be positive. In addition, $\sigma(\kappa)>0$ only when $\Delta>\left(1+d_{2}\right)^{2}$. This occurs whenever $\gamma_{12} \gamma_{21} \operatorname{sinc}^{2}(\kappa \delta)>d_{2}$. Since the maximum value of $\operatorname{sinc}^{2}(\kappa \delta)$ is 1 , which is achieved at $\kappa=0$, we arrive at the following necessary criterion for pattern formation, which is also sufficient if we either drop the boundary conditions or take the $\delta \rightarrow 0$ limit 


$$
\gamma_{12} \gamma_{21}>d_{2}
$$

Furthermore, any patterns that do form are stationary patterns, since the eigenvalues are always real if their real part is positive.

There are three distinct biologically relevant situations, which correspond to different values of $\gamma_{12}$ and $\gamma_{21}$, as follows

1. Mutual avoidance: $\gamma_{12}, \gamma_{21}<0$

2. Mutual attraction: $\gamma_{12}, \gamma_{21}>0$

3. Pursue-and-avoid: $\gamma_{12}<0<\gamma_{21}$ or $\gamma_{21}<0<\gamma_{12}$

There are also the edge cases where $\gamma_{12}=0$ or $\gamma_{21}=0$, which we will not focus on. Notice that the 'pursue-and-avoid' case cannot lead to the emergence of patterns (Fig. 2e), as it is inconsistent with the inequality in (21). However, the other two situations can.

Mutual avoidance leads to spatial segregation if Inequality (21) is satisfied (Fig. 2c). Some previous models of territory formation in animal populations by the present authors have a very similar form to the mutual avoidance model here, so we refer to Potts and Lewis (2016a, b) for details of this situation. Mutual attraction leads to aggregation of both populations in a particular part of space, whose width roughly corresponds to the width of the spatial averaging kernel, $(x-\delta, x+\delta)$ (Fig. 2a), as long as Inequality (21) is satisfied.

The characterisation of between-population movement responses into "mutual avoidance', 'mutual attraction', and 'pursue-and-avoid' enables us to categorise examples of the system in Eqs. $(12,14,15)$ by means of a simple schematic diagram. We construct one node for each population, ensuring that no three distinct nodes are in a straight line. Then, an arrow is added from node $i$ to node $j$ if $\gamma_{i j}>0$. If $\gamma_{i j}<0$, an arrow is added from node $i$ in the direction anti-parallel to the line from node $i$ to node $j$. These diagrams allow us to see quickly the qualitative relationship between the populations (see Fig. 2b,d,f for the $N=2$ case and Fig. 4 b for some examples in the $N=3$ case).

\subsection{An Energy Functional Approach to Analysing Patterns}

We can gain qualitative understanding of the patterns observed in Fig. 2a-d via use of an energy functional approach, by assuming $\gamma_{1,2}=\gamma_{2,1}=\gamma$, and $d_{2}=1$. In particular, this approach gives a mathematical explanation for the appearance of aggregation patterns when $\gamma>0$ and segregation patterns when $\gamma<0$. The results rely on the assumption that, for all $i, u_{i}(x, 0)>0$ implies $u_{i}(x, t)>0$ for all $t$, which can be shown by the application of a comparison theorem to Eqs. $(8,13)$, assuming $\partial A_{i}(x) / \partial x$ is bounded. Throughout this section, our spatial coordinates will be defined on the quotient space $[0,1] /\{0,1\}$, which is consistent with our use of periodic boundary conditions. 

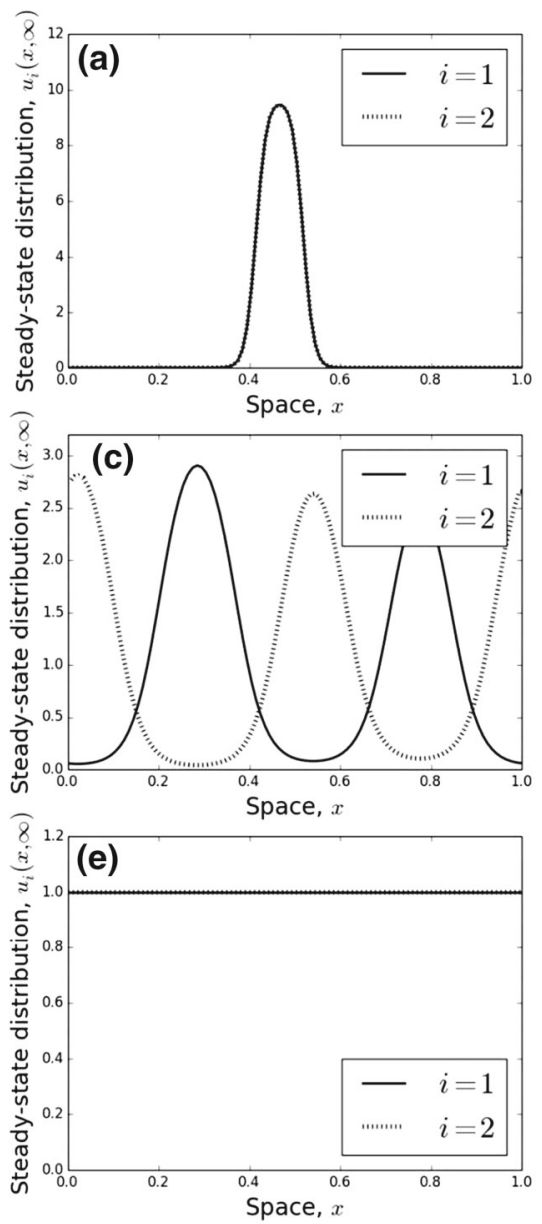

(b) Mutual attraction

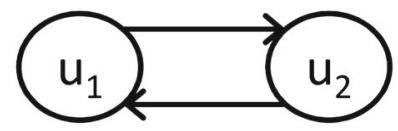

(d) Mutual avoidance
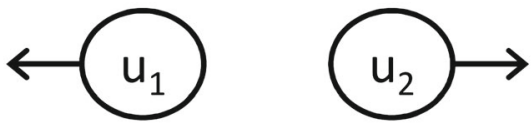

(f) Pursue-and-avoid

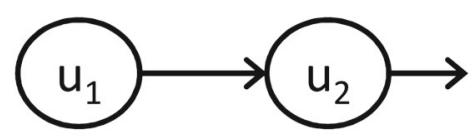

Fig. 2 Dynamics for a two-species system. Here, there are three cases: mutual attraction, mutual avoidance, and pursue-and-avoid. a The steady state of a model of mutual attraction, with $\gamma_{12}=\gamma_{21}=2$, and $\delta=0.1$, with a schematic of this situation in b. $\mathbf{c}$ The steady state of a mutual avoidance model with $\gamma_{12}=\gamma_{21}=-2$, and $\delta=0.1$, with corresponding schematic in $\mathbf{d}$. e The steady state of a pursue-and-avoid model (where patterns never form) with $\gamma_{12}=2, \gamma_{21}=-2$ and $\delta=0.1$, with corresponding schematic in $\mathbf{f}$

Our method makes use of the following formulation of Eq. (12)

$$
\frac{\partial u_{i}}{\partial t}=\frac{\partial}{\partial x}\left[u_{i} \frac{\partial}{\partial x}\left(d_{i} \ln \left(u_{i}\right)-\sum_{j \neq i} \gamma_{i j} \mathcal{K} * u_{j}\right)\right],
$$

and also the energy functional

$$
E\left(u_{1}, u_{2}\right)=\int_{0}^{1}\left\{u_{1}\left[2 \ln \left(u_{1}\right)-\gamma \mathcal{K} * u_{2}\right]+u_{2}\left[2 \ln \left(u_{2}\right)-\gamma \mathcal{K} * u_{1}\right]\right\} \mathrm{d} x,
$$


where $\mathcal{K}(x)$ is a bounded function (i.e. $\|\mathcal{K}\|_{\infty}<\infty$ ), symmetric about $x=0$ on the domain $[0,1] /\{0,1\}$, with $\|\mathcal{K}\|_{1}=1$, and $*$ denotes the following spatial convolution

$$
\mathcal{K} * u_{i}(x, t)=\int_{0}^{1} \mathcal{K}(x-y) u_{i}(y, t) \mathrm{d} y .
$$

In our situation, Eq. (14) implies that $\mathcal{K}(x)=1 /(2 \delta)$ for $-\delta<x<\delta(\bmod 1)$ and $\mathcal{K}(x)=0$ for $\delta \leq x \leq 1-\delta$. We consider solutions $u_{1}(x, t)$ and $u_{2}(x, t)$ that are continuous functions of $x$ and $t$.

We show that the energy functional from Eq. (23) decreases over time to a minimum, which represents the steady-state solution of the system. The monotonic decrease of $E$ over time is shown as follows

$$
\begin{aligned}
\frac{\partial E}{\partial t}= & \int_{0}^{1}\left\{\frac{\partial u_{1}}{\partial t}\left[2 \ln \left(u_{1}\right)-\gamma \mathcal{K} * u_{2}\right]+\frac{\partial u_{2}}{\partial t}\left[2 \ln \left(u_{2}\right)-\gamma \mathcal{K} * u_{1}\right]\right\} \mathrm{d} x \\
& +\int_{0}^{1}\left[2 \frac{\partial u_{1}}{\partial t}+2 \frac{\partial u_{2}}{\partial t}-\gamma u_{1} \mathcal{K} * \frac{\partial u_{2}}{\partial t}-\gamma u_{2} \mathcal{K} * \frac{\partial u_{1}}{\partial t}\right] \mathrm{d} x \\
= & \int_{0}^{1}\left\{2 \frac{\partial u_{1}}{\partial t}+2 \frac{\partial u_{2}}{\partial t}+\frac{\partial u_{1}}{\partial t}\left[2 \ln \left(u_{1}\right)-2 \gamma \mathcal{K} * u_{2}\right]+\frac{\partial u_{2}}{\partial t}\left[2 \ln \left(u_{2}\right)-2 \gamma \mathcal{K} * u_{1}\right]\right\} \mathrm{d} x \\
= & 2 \int_{0}^{1}\left\{\frac{\partial}{\partial x}\left[u_{1} \frac{\partial}{\partial x}\left(\ln \left(u_{1}\right)-\gamma \mathcal{K} * u_{2}\right)\right]\left[1+\ln \left(u_{1}\right)-\gamma \mathcal{K} * u_{2}\right]\right. \\
& \left.+\frac{\partial}{\partial x}\left[u_{2} \frac{\partial}{\partial x}\left(\ln \left(u_{2}\right)-\gamma \mathcal{K} * u_{1}\right)\right]\left[1+\ln \left(u_{2}\right)-\gamma \mathcal{K} * u_{1}\right]\right\} \mathrm{d} x \\
= & 2\left[u_{1} \frac{\partial}{\partial x}\left(\ln \left(u_{1}\right)-\gamma \mathcal{K} * u_{2}\right)\left(1+\ln \left(u_{1}\right)-\gamma \mathcal{K} * u_{2}\right)\right. \\
& \left.+u_{2} \frac{\partial}{\partial x}\left(\ln \left(u_{2}\right)-\gamma \mathcal{K} * u_{1}\right)\left(1+\ln \left(u_{2}\right)-\gamma \mathcal{K} * u_{1}\right)\right]_{0}^{1} \\
& -2 \int_{0}^{1}\left\{\left[u_{1} \frac{\partial}{\partial x}\left(\ln \left(u_{1}\right)-\gamma \mathcal{K} * u_{2}\right)\right] \frac{\partial}{\partial x}\left(\ln \left(u_{1}\right)-\gamma \mathcal{K} * u_{2}\right)\right. \\
& \left.+\left[u_{2} \frac{\partial}{\partial x}\left(\ln \left(u_{2}\right)-\gamma \mathcal{K} * u_{1}\right)\right] \frac{\partial}{\partial x}\left(\ln \left(u_{2}\right)-\gamma \mathcal{K} * u_{1}\right)\right\} \mathrm{d} x \\
= & -2 \int_{0}^{1}\left\{\left[u_{1} \frac{\partial}{\partial x}\left(\ln \left(u_{1}\right)-\gamma \mathcal{K} * u_{2}\right)\right] \frac{\partial}{\partial x}\left(\ln \left(u_{1}\right)-\gamma \mathcal{K} * u_{2}\right)\right. \\
& \left.+\left[u_{2} \frac{\partial}{\partial x}\left(\ln \left(u_{2}\right)-\gamma \mathcal{K} * u_{1}\right)\right] \frac{\partial}{\partial x}\left(\ln \left(u_{2}\right)-\gamma \mathcal{K} * u_{1}\right)\right\} \mathrm{d} x \\
= & -2 \int_{0}^{1}\left\{u_{1}\left[\frac{\partial}{\partial x}\left(\ln \left(u_{1}\right)-\gamma \mathcal{K} * u_{2}\right)\right]^{2}+u_{2}\left[\frac{\partial}{\partial x}\left(\ln \left(u_{2}\right)-\gamma \mathcal{K} * u_{1}\right)\right] \mathrm{d} x\right. \\
2 & (25) \\
&
\end{aligned}
$$

Here, the first equality uses Eq. (23), the second uses the fact that $\int_{0}^{1} f(x) \mathcal{K} * h(x) \mathrm{d} x=$ $\int_{0}^{1} h(x) \mathcal{K} * f(x) \mathrm{d} x$ as long as $\mathcal{K}(x)$ is symmetric about 0 in $[0,1] /\{0,1\}$, and also requires that $\gamma_{1,2}=\gamma_{2,1}=\gamma$, the third uses Eq. (22), the fourth is integration by parts, 
the fifth uses the fact that $u_{i}(0)=u_{i}(1)$ and $\mathcal{K} * u_{i}(0)=\mathcal{K} * u_{i}(1)$ for $i \in\{1,2\}$ (i.e. periodic boundary conditions, Eq. 13), the sixth is just a rearrangement, and the inequality at the end uses the fact that $u_{i}(x, t)>0$ for all $i, x, t$. In all, Eq. (25) shows that $E\left(u_{1}, u_{2}\right)$ is decreasing over time. The following shows that $E\left(u_{1}, u_{2}\right)$ is bounded below

$$
\begin{aligned}
E\left(u_{1}, u_{2}\right) & =2 \int_{0}^{1}\left[u_{1} \ln \left(u_{1}\right)+u_{2} \ln \left(u_{2}\right)\right] \mathrm{d} x-\int_{0}^{1}\left[u_{1} \mathcal{K} * u_{1}+u_{2} \mathcal{K} * u_{2}\right] \mathrm{d} x \\
& \geq-4 \mathrm{e}^{-1}-\int_{0}^{1}\left[u_{1} \mathcal{K} * u_{1}+u_{2} \mathcal{K} * u_{2}\right] \mathrm{d} x \\
& \geq-4 \mathrm{e}^{-1}-\left\|u_{1}\right\|_{1}\left\|\mathcal{K} * u_{1}\right\|_{\infty}-\left\|u_{2}\right\|_{1}\left\|\mathcal{K} * u_{2}\right\|_{\infty} \\
& \geq-4 \mathrm{e}^{-1}-\left\|u_{1}\right\|_{1}\|\mathcal{K}\|_{\infty}\left\|u_{1}\right\|_{1}-\left\|u_{2}\right\|_{1}\|\mathcal{K}\|_{\infty}\left\|u_{2}\right\|_{1} \\
& \geq-4 \mathrm{e}^{-1}-2\|\mathcal{K}\|_{\infty}
\end{aligned}
$$

Here, the first inequality uses the fact that $\inf _{u_{i} \geq 0}\left\{u_{i} \ln \left(u_{i}\right)\right\}=-\mathrm{e}^{-1}$, the second uses Hölder's inequality, the third uses Young's inequality, and the fourth uses the fact that $\left\|u_{1}\right\|_{1}=1$ (Eq. 15). For the absence of doubt, the definition $\|f\|_{p}=$ $\left(\int_{0}^{1}|f(x, t)|^{p} \mathrm{~d} x\right)^{1 / p}$, for $p \in[1, \infty]$, is used throughout (26). Again, note that the inequality $u(x, t)>0$ is required for the sequence of inequalities in (26) to hold.

The inequalities in (25) and (26) together demonstrate that $E\left(u_{1}, u_{2}\right)$ moves towards a minimum as $t \rightarrow \infty$, which is given at the point where $\frac{\partial E}{\partial t}=0$. The latter equation is satisfied when the following two conditions hold

$$
\begin{aligned}
& \ln \left(u_{1}\right)-\gamma \mathcal{K} * u_{2}=\eta_{1}, \\
& \ln \left(u_{2}\right)-\gamma \mathcal{K} * u_{1}=\eta_{2},
\end{aligned}
$$

where $\eta_{1}$ and $\eta_{2}$ are constants.

Equations (27-28) can be used to give qualitative properties of the long-term distribution of the system in Eqs. $(12,14,15)$ for $N=2$ and $\gamma_{1,2}=\gamma_{2,1}=\gamma$. First, by differentiating Eqs. (27-28) with respect to $x$, we find that

$$
\begin{aligned}
& \frac{\partial u_{1}}{\partial x} \frac{1}{u_{1}}=\gamma \frac{\partial}{\partial x}\left(\mathcal{K} * u_{2}\right), \\
& \frac{\partial u_{2}}{\partial x} \frac{1}{u_{2}}=\gamma \frac{\partial}{\partial x}\left(\mathcal{K} * u_{1}\right) .
\end{aligned}
$$

Thus, $\gamma>0$ implies that $\frac{\partial u_{1}}{\partial x}$ has the same sign as $\frac{\partial}{\partial x}\left(\mathcal{K} * u_{2}\right)$ so any patterns that may form will be aggregation patterns (Fig. 2a, b). Furthermore, $\gamma<0$ implies that $\frac{\partial u_{1}}{\partial x}$ has the opposite sign to $\frac{\partial}{\partial x}\left(\mathcal{K} * u_{2}\right)$ so any patterns that form will be segregation patterns (Fig. 2c, d).

Second, by making the following moment closure approximation

$$
\mathcal{K} * u_{i} \approx u_{i}+\sigma^{2} \frac{\partial^{2} u_{i}}{\partial x^{2}},
$$


where $\sigma^{2}$ is the variance of $\mathcal{K}(x)$, we can gain insight by examining the plot of $\frac{\partial^{2} u_{i}}{\partial x^{2}}$ against $u_{i}$ in particular cases. To give an example in the case of aggregation, if $u_{1} \approx u_{2}$ (as in Fig. 2a), then we have $\gamma>0$ by Eqs. (29-30). Equation (28) implies

$$
\sigma^{2} \frac{\partial^{2} u_{1}}{\partial x^{2}} \approx \frac{1}{\gamma}\left[\ln \left(u_{1}\right)-\eta_{2}\right]-u_{1} .
$$

The right-hand side of Eq. (32) has a unique maximum, which is above the horizontal axis as long as $\eta_{2}<-1-\ln (\gamma)$ (Fig. 3a). In this case, there are two numbers $a, b \in \mathbb{R}_{>0}$ such that $\frac{\partial^{2} u_{i}}{\partial x^{2}}>0$ when $a<u_{i}<b$ and $\frac{\partial^{2} u_{i}}{\partial x^{2}}<0$ for $u_{i}<a$ or $u_{i}>b$. A
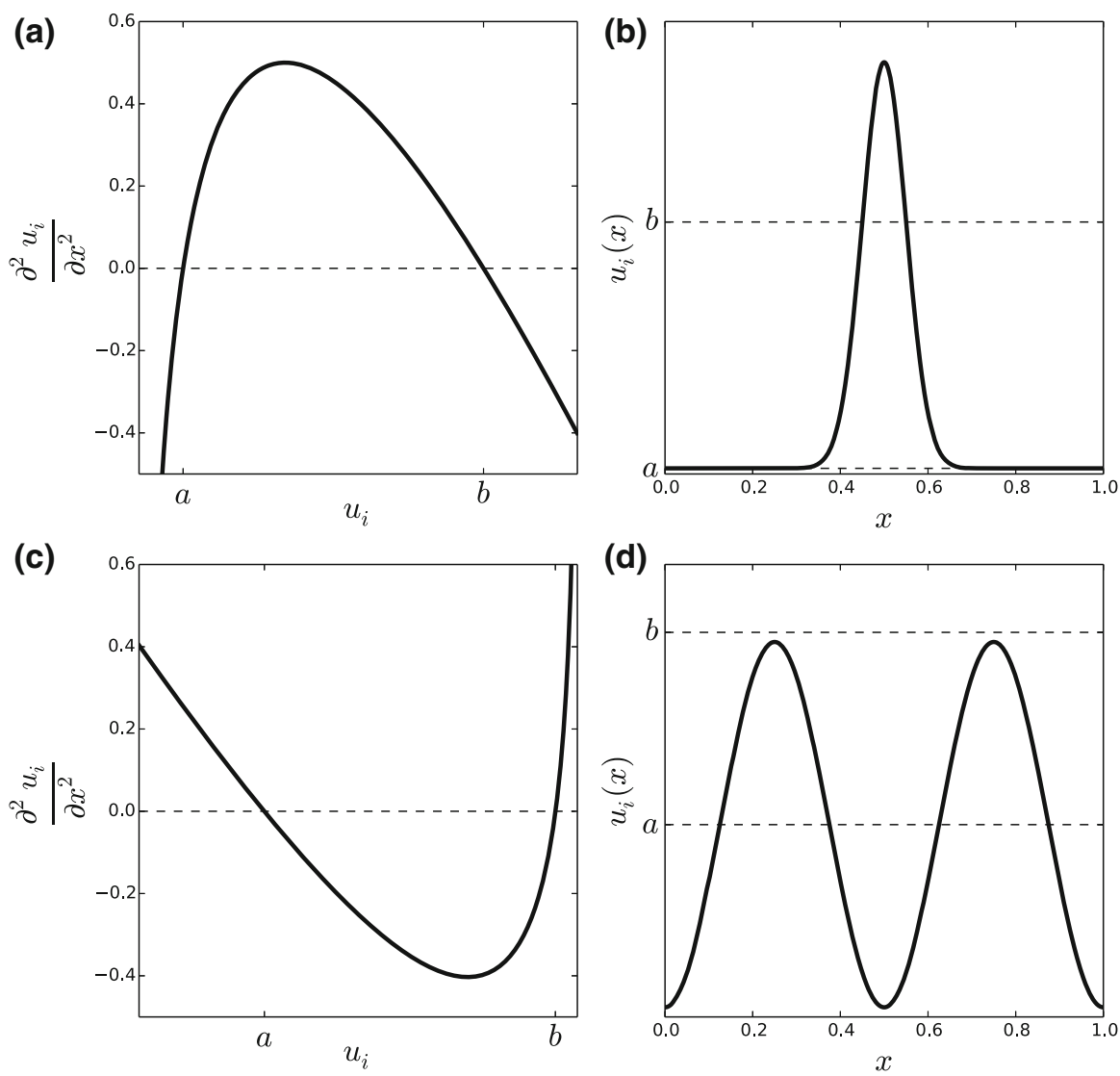

Fig. 3 Understanding the patterns from Fig. 2 using energy functionals. a An example of $\frac{\partial^{2} u_{i}}{\partial x^{2}}$ as a function of $u_{i}$ (Eq. 32) when the energy is minimised (Eqs. 27-28) and the moment closure approximation from Eq. (31) is applied, for the aggregation case, $u_{1} \approx u_{2}$. We see that $\frac{\partial^{2} u_{i}}{\partial x^{2}}$ is positive for $a<u_{i}<b$ and negative when $u_{i}<a$ or $u_{i}>b$. There are various possible smooth solutions, $u_{i}(x, \infty)$, that satisfy this property. b An example corresponding qualitatively to Fig. 2a. c, d Are analogous to a and b, respectively, but for the situation where we have segregation, so $u_{1} \approx 2-u_{2}$. Note that $\mathbf{d}$ qualitatively resembles Fig. $2 \mathrm{c}$ 
possible curve that satisfies this property is given in Fig. $3 \mathrm{~b}$ and qualitatively resembles Fig. 2a.

To give an example in the case of segregation $(\gamma<0)$, suppose that $u_{1} \approx 2-u_{2}$. Then, by a similar argument to the $u_{1} \approx u_{2}$ case, $\frac{\partial^{2} u_{i}}{\partial x^{2}}$ has a unique minimum as long as $\eta_{2}<-1-\ln (-\gamma)-2 \gamma$. In this case, there are two numbers $a, b \in \mathbb{R}$ such that $\frac{\partial^{2} u_{i}}{\partial x^{2}}<0$ when $a<u_{i}<b$ and $\frac{\partial^{2} u_{i}}{\partial x^{2}}>0$ for $u_{i}<a$ or $u_{i}>b$. A possible curve that satisfies this property is given in Fig. $3 \mathrm{~d}$ and qualitatively resembles Fig. 2c.

\section{The Case of Three Interacting Populations $(N=3)$}

Although the $N=2$ case only allows for stationary pattern formation [often called a Turing instability after Turing (1952)], for $N>2$ we can observe both stationary and oscillating patterns. The latter arise from what is sometimes known as a wave instability, where the dominant eigenvalue of $M(\kappa, \delta)$ is not real but has positive real part, for some $\kappa$. For $N>2$, the situation becomes too complicated for analytic expressions of the eigenvalues to give any meaningful insight [and indeed, these expressions cannot be found for $N>4$ by a classical result of Galois Theory; see Stewart (2015)], so we begin by examining the eigenvalues for certain example cases in the limit $\delta \rightarrow 0$. This involves finding eigenvalues of the matrix $M_{0}$ given in Eq. (18).

Figure 4 gives an example of how (i) stationary patterns, (ii) oscillatory patterns, and (iii) no patterns can emerge in different regions of parameter space when $N=3$. Here, we have fixed all the $\gamma_{i j}$ except $\gamma_{12}$ and $\gamma_{23}$. Specifically, $d_{2}=d_{3}=\gamma_{21}=\gamma_{31}=$ $\gamma_{32}=1$, and $\gamma_{13}=-1$. When $\gamma_{12}<0<\gamma_{23}$, this corresponds to a mutual attraction between populations 2 and 3 with both 2 and 3 pursuing 1 in a pursue-and-avoid

(a)

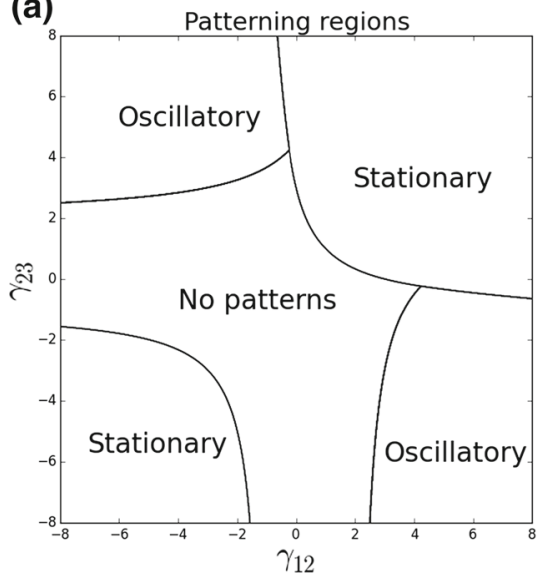

(b)

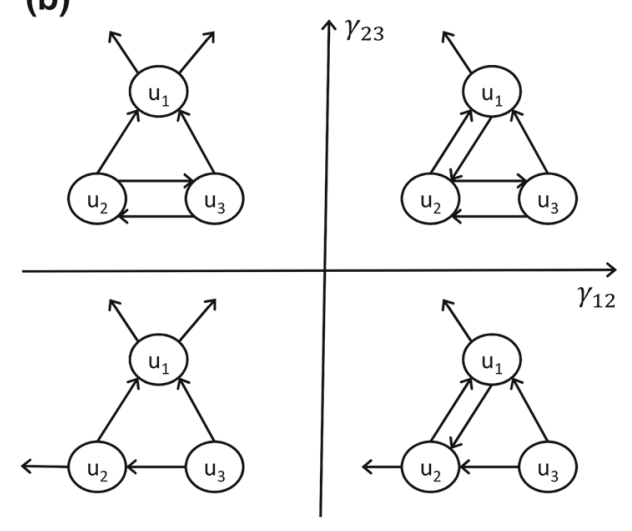

Fig. 4 Dynamics for example three-species systems. a The pattern formation regions, as predicted by linear analysis, for the system in Eqs. $(12,14,15)$ in the case $N=3$, where $d_{2}=d_{3}=\gamma_{21}=\gamma_{31}=\gamma_{32}=1$, $\gamma_{13}=-1$, and $\gamma_{12}, \gamma_{23}$ are varied. b The schematic diagrams of the systems, corresponding to the four quadrants of $\left(\gamma_{12}, \gamma_{23}\right)$-space 
situation (Fig. 4b, top-left). When $\gamma_{12}, \gamma_{23}>0,3$ is pursuing 1 in a pursue-and-avoid, whilst 2 is mutually attracted to both 1 and 3 (Fig. 4b, top-right). If $\gamma_{23}<0<\gamma_{12}, 3$ is pursuing both 1 and 2 in a pursue-and-avoid, whilst 1 and 2 are mutually attracting (Fig. 4b, bottom right). Finally, if $\gamma_{12}, \gamma_{23}<0$, then 3 is pursuing both 1 and 2 in a pursue-and-avoid and 2 is pursuing 1 in a pursue-and-avoid (Fig. 4b, bottom left).

We solved the system in Eqs. (12-15) for various examples from both the stationary and oscillatory pattern regimes shown in Fig. 4. For this, we used periodic boundary conditions as in Eq. (13). We used a finite difference method, coded in Python, with a spatial granularity of $h=10^{-2}$ and a temporal granularity of $\tau=10^{-5}$. Initial conditions were set to be small random fluctuations from the spatially homogeneous steady state.

Stationary patterns can give rise to space partitioned into different areas for use by different populations (Fig. 5, Supplementary Video SV1), with differing amounts of

(a)

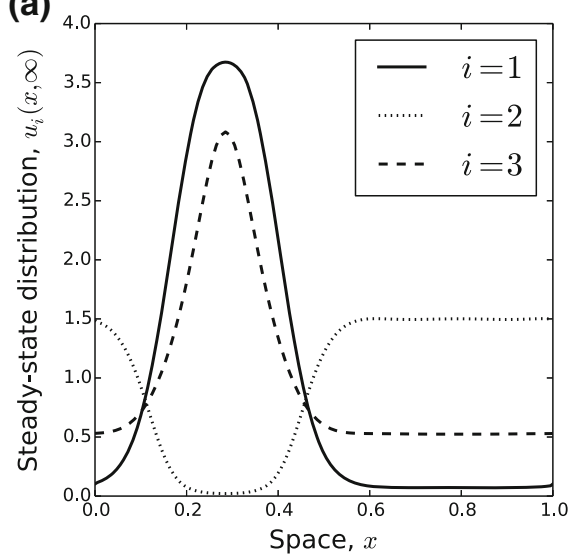

(c)

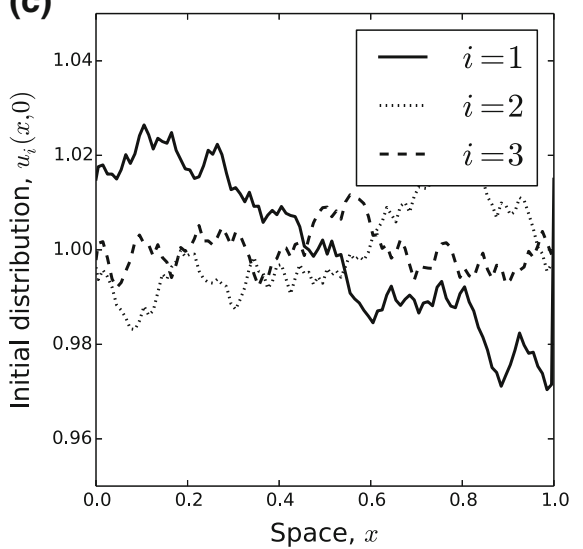

(b)

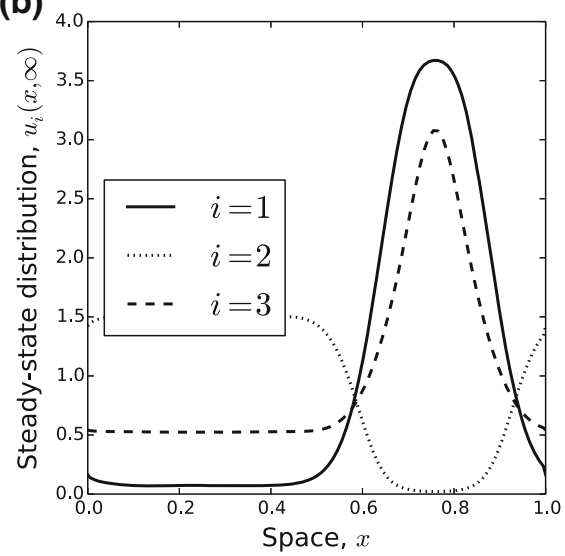

(d)

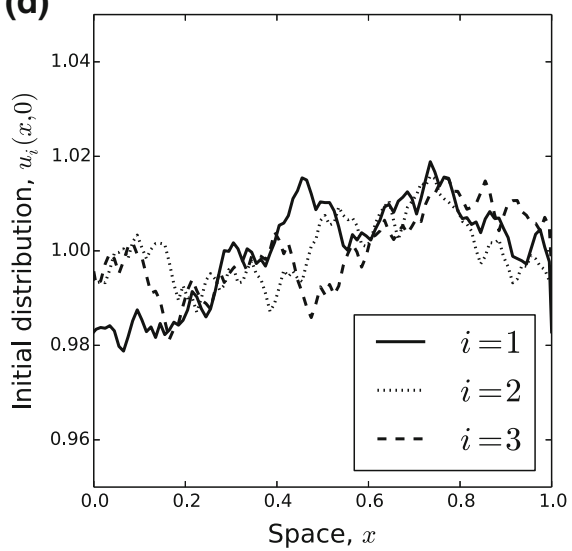

Fig. 5 Example three-species systems with stationary distributions. a, b Two stable steady-state distributions for the system in $(12,14,15)$ in the case $N=3$, where $d_{2}=d_{3}=\gamma_{21}=\gamma_{31}=\gamma_{32}=1, \gamma_{13}=-1$, $\gamma_{12}=\gamma_{23}=-4$, and $\delta=0.1$. c [resp. (d)] The initial condition that led to the stationary distribution in a [resp. (b)] 
overlap. Interestingly, the precise location of the segregated regions depends upon the initial conditions (compare panels (a) and (b) in Fig. 5), but the rough size of the regions appears to be independent of the initial condition (at least for the parameter values we tested). Considering the abundance of individuals as a whole (i.e. $u_{1}+u_{2}+u_{3}$ ), notice that certain regions of space emerge that contain more animals than others. This is despite the fact that there is no environmental heterogeneity in the model.

The extent to which populations use the same parts of space depends upon the strength of attraction or repulsion. In Fig. 5a, b, the demarcation between populations 1 and 2 is quite stark, owing to the strong avoidance of population 2 by population 1 $\left(\gamma_{12}=-4\right)$ and a relatively small attraction of population 2 to population $1\left(\gamma_{21}=1\right)$, whereas, although population 1 seeks to avoid 3, the strength of avoidance is smaller $\left(\gamma_{13}=-1\right)$, but the attraction of population 3 to population 1 is of a similar magnitude $\left(\gamma_{31}=1\right)$. Therefore, populations 1 and 3 overlap considerably.

Oscillatory patterns can be quite complex (Supplementary Video SV2), varying from situations where there appear to be periodic oscillations (Fig. 6a) to those where the periodicity is much less clear (Fig. 6b). To understand their behaviour, we use a method of numerical bifurcation analysis adapted from Painter and Hillen (2011). This method begins with a set of parameters in the region of no pattern formation but close to the region of oscillatory patterns. In particular, we choose parameter values

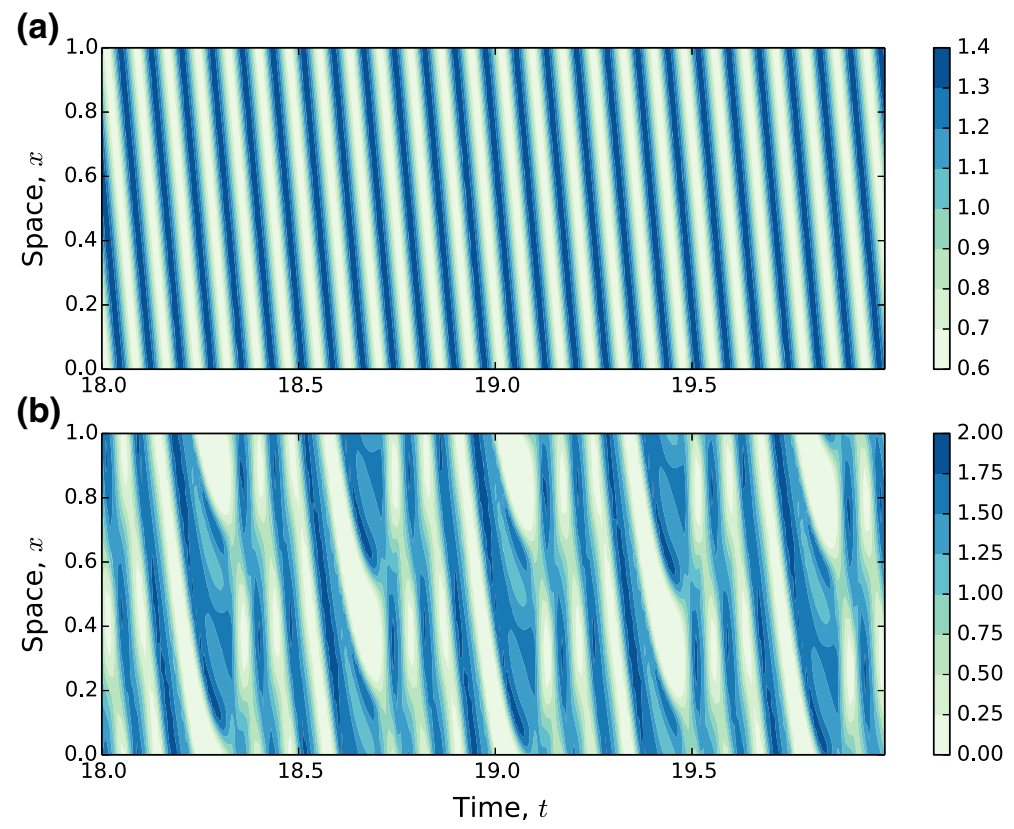

Fig. 6 Example three-species systems with oscillatory distributions. Here, we show the change in $u_{1}(x, t)$ over space and time for two sets of parameter values. Both panels have parameter values identical to the fixed parameters from Fig. 4 a, additionally fixing $\gamma_{23}=-4$ and $\delta=0.1$. In a, we have $\gamma_{12}=3.3$ and $\mathbf{b}$ has $\gamma_{12}=4$. We started with random initial conditions and then ran the system to (dimensionless) time $t=20$. The plots display values of $u_{1}(x, t)$ for $x \in[0,1]$ and $t \in[18,20]$. Plots for $t \in[14,16]$ and $t \in[16,18]$ (not shown) are very similar, indicating that the system has reached its attractor (color figure online) 
identical to the fixed values for Fig. 4 a (i.e. $d_{2}=d_{3}=\gamma_{21}=\gamma_{31}=\gamma_{32}=1$, $\gamma_{13}=-1$ ) and also $\gamma_{23}=-2.5$ and $\gamma_{12}=3$. We then perform the following iterative procedure:

1. Solve the system numerically until $t=10$, by which time the attractor has been reached,

2. Increment $\gamma_{12}$ by a small value (we used 0.005 ) and set the initial conditions for the next iteration to be the final values of $u_{1}(x, t), u_{2}(x, t)$, and $u_{3}(x, t)$ from the present numerical solution.

This method is intended to approximate a continuous bifurcation analysis. To analyse the resulting patterns, we focus on the value of the system for a fixed point $x=0.5$ and examine how attractor of the system $\left(u_{1}(0.5, t), u_{2}(0.5, t), u_{3}(0.5, t)\right)$ changes as increase $\gamma_{12}$ into the region of oscillatory patterns.

Figure 7 shows these attractors for various $\gamma_{12}$ values. First, we observe a small loop appearing just after the system goes through the bifurcation point (Fig. 7a). This loop then grows (Fig. $7 b, c)$ and, when $\gamma_{12} \approx 4.1$, undergoes a period-doubling bifurcation (Fig. 7d). The attractor remains as a double-period loop (Fig. 7e, f) until $\gamma_{12} \approx 5.77$ where it doubles again (Fig. $7 \mathrm{~g}, \mathrm{~h}$ ). Such a sequence of period doubling is a hallmark of a chaotic system. Indeed, as $\gamma_{12}$ is increased further, the patterns cease to have obvious period patterns (Fig. 7i) and gain a rather more irregular look, suggestive of chaos.

\section{Discussion}

We have used a class of diffusion-taxis systems for analysing the effect of betweenpopulation movement responses on spatial distributions of these populations. Our models are sufficient for incorporating taxis effects due to both direct and indirect animal interactions, so are of general use for a wide range of ecological communities. We have shown that spatial patterns in species distributions can emerge spontaneously as a result of these interactions. What is more, these patterns may not be fixed in time, but could be in constant flux. This brings into question the implicit assumption behind many species distribution models that the spatial distribution of a species in a fixed environment is roughly stationary over time.

Mathematically, our approach builds upon recent diffusion-taxis models of territory formation (Potts and Lewis 2016a, b). However, these latter models only consider two populations, and only in the case where there is mutual avoidance (i.e. Fig. 2c, d). We have shown that, when there is just one more population in the mix $(N=3)$, the possible patterns that emerge can be extremely rich, incorporating stationary patterns, periodic oscillations, and irregular patterns that may be chaotic. Although irregular and chaotic spatio-temporal patterns have been observed in spatial predator-prey systems (Sherratt et al. 1995, 1997), this is one of the few times they have been discovered as arising from inter-population avoidance models (but see White et al. 1996, Section 8.2). These possibilities will extend to the situation of $N>3$, which is typical of most real-life ecosystems (e.g. Vanak et al. 2013). 

(a)
$\gamma_{12}=3.5$

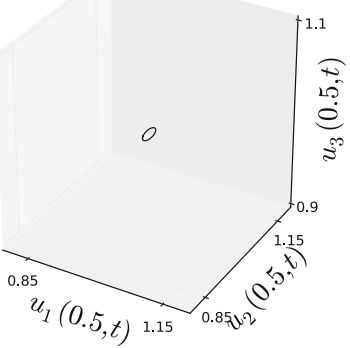

(d)

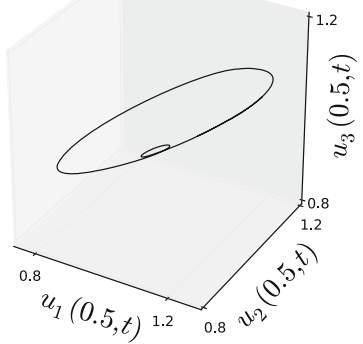

(g)

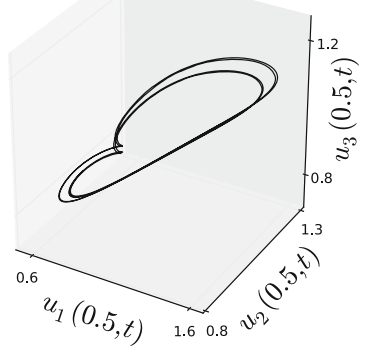

(b) $\quad \gamma_{12}=3.55$

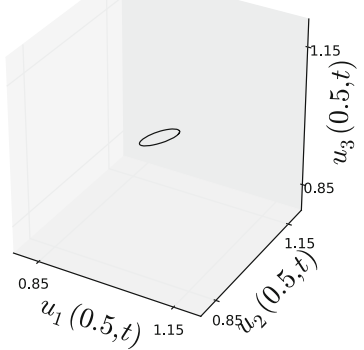

(e)

$\gamma_{12}=5.1$

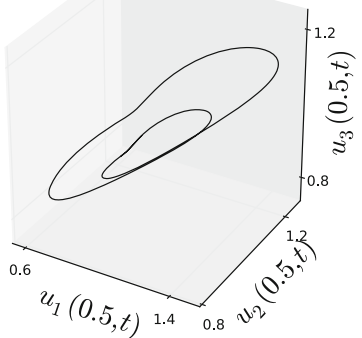

(h)

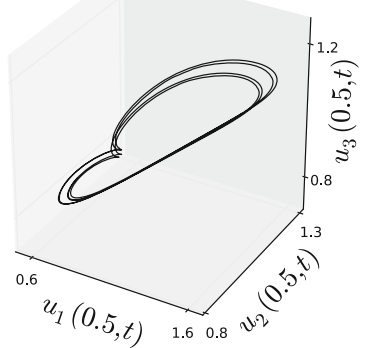

(c) $\quad \gamma_{12}=3.61$

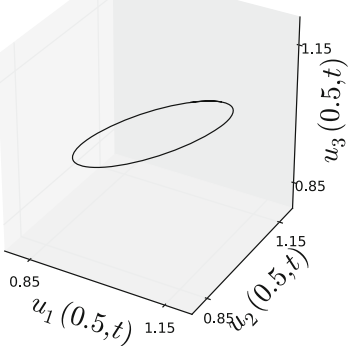

(f) $\gamma_{12}=5.6$

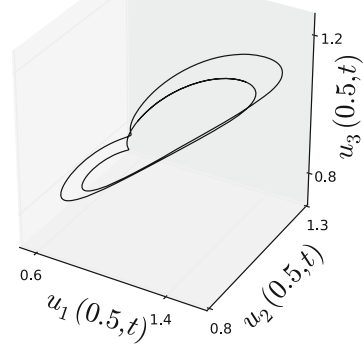

(i) $\gamma_{12}=5.85$

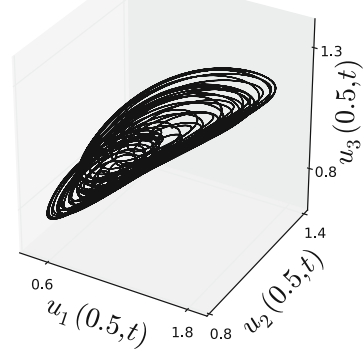

Fig. 7 Numerical bifurcation analysis. This sequence of plots shows the attractors just after the system passes through a bifurcation point from a region of no patterns to one of oscillatory patterns. Each panel shows the locus of the point $\left(u_{1}(0.5, t), u_{2}(0.5, t), u_{3}(0.5, t)\right)$ as time changes for a particular set of parameter values. In all panels, $d_{2}=d_{3}=\gamma_{21}=\gamma_{31}=\gamma_{32}=1, \gamma_{13}=-1$, and $\gamma_{23}=-2.5$. The value of $\gamma_{12}$ increases from $\mathbf{a}$ to $\mathbf{i}$ and is given in the panel title. As $\gamma_{12}$ increases, we observe a sequence of period-doubling bifurcations leading to irregular patterns, suggestive of a chaotic system

The models studied here are closely related to aggregation models, which are well studied, often with applications to cell biology in mind (Alt 1985; Mogilner and Edelstein-Keshet 1999; Topaz et al. 2006; Painter et al. 2015). In these models, populations exhibit self-attraction alongside diffusion and are usually framed with just a single population in mind [although some incorporate more, e.g. Painter et al. (2015), Burger et al. (2018)]. In contrast to our situation, this self-attraction process can enable spontaneous aggregation to occur in a single population. Similar to our situation, in 
these self-attraction models it is typical to observe ill-posed problems unless some form of regularisation is in place, either through non-local terms (Mogilner and EdelsteinKeshet 1999; Briscoe et al. 2002; Topaz et al. 2006) or other means such as mixed spatio-temporal derivatives (Padrón 1998).

We have decided not to incorporate self-attraction into our framework. This is both for simplicity of analysis and because the animal populations we have in mind will tend to spread on the landscape in the absence of interactions, so are well described using diffusion as a base model (Okubo and Levin 2013; Lewis et al. 2016). However, in principle it is a simple extension to incorporate self-interaction into out framework, simply by dropping the $j \neq i$ restriction in Eq. (12). Indeed, for $N=2$, very similar models have been studied for aggregation/segregation properties (Burger et al. 2018) and pattern formation (Painter et al. 2015). In those studies, a combination of self-attraction and pursue-and-avoid can, contrary to the pure pursue-and-avoid case studied here, lead to moving spatial patterns where one aggregated population (the avoiders) leads the other one (the pursuers) in a 'chase' across the landscape (Painter 2009), a phenomenon observed in certain cell populations (Theveneau et al. 2013). For $N>2$, however, we have shown that the story regarding spatial patterns can already be very rich and complicated without self-attraction, so understanding the effect of this extra complication would be a formidable exercise.

Another natural extension of our work, from a mathematical perspective, would be to add reaction terms (a.k.a. kinetics) into our model, accounting for deaths (e.g. due to predation or as a result of competition) and births, by adding a function $f_{i}\left(u_{1}, \ldots, u_{N}\right)$ to Eq. (12) for each $i$. Biologically, this would change the timescale over which our model is valid, since in the present study we have explicitly set out to model timescales over which where births and deaths are negligible. Nonetheless, this extension is worthy of discussion since the addition of such terms leads to a class of so-called cross-diffusion models, which are well studied (Shigesada et al. 1979; Gambino et al. 2009; Shi et al. 2011; Tania et al. 2012; Potts and Petrovskii 2017). The term 'crossdiffusion' has been used in various guises, but the general form can incorporate both taxis terms of the type described here, as well as other terms that model various movement responses between populations. These cross-diffusion terms can combine with the reaction terms to drive pattern formation (Shi et al. 2011; Tania et al. 2012), as well as altering spreading speeds (Gambino et al. 2009; Girardin and Nadin 2015), and the outcome of competitive dynamics (Potts and Petrovskii 2017). The key difference between our work and traditional studies of cross-diffusion is that rich patterns form in our model despite the lack of kinetics. As such, we separate out the effect of taxis on pattern formation from any interaction with the reaction terms.

Our mathematical insights suggest that there is an urgent need to understand the extent to which the underlying movement processes in our model are prevalent in empirical ecosystems. Much effort is spent in understanding species distributions (Manly et al. 2002; Araujo and Guisan 2006; Jiménez-Valverde et al. 2008), often motivated by highly applied questions such as understanding the effect of climate change on biodiversity loss (Gotelli and Stanton-Geddes 2015), planning conservation efforts (Rodríguez et al. 2007; Evans et al. 2015), and mitigating negative effects of disease spread (Fatima et al. 2016) and biological invasions (Mainali et al. 2015). Species distribution models typically seek to link the distribution of species with envi- 
ronmental covariates, whereas the effect of between-population movement responses is essentially ignored. Presumably, this is because it is considered as 'noise' that likely averages out over time. In contrast, this study suggests that the patterns emerging from between-population movements may be fundamental drivers of both transient and asymptotic species distributions.

Fortuitously, recent years have seen the development of techniques for measuring the effects of foreign populations on animal movement. Animal biologging technology has become increasingly smaller, cheaper, and able to gather data at much higher frequencies than ever before (Wilmers et al. 2015; Williams et al., in review). Furthermore, statistical techniques have become increasingly refined to uncover the behavioural mechanisms behind animals' movement paths (Albertsen et al. 2015; Avgar et al. 2016; Michelot et al. 2016; Potts et al. 2018). In particular, these include inferring interactions between wild animals, both direct (Vanak et al. 2013) and mediated by environmental markers (Latombe et al. 2014; Potts et al. 2014).

Consequently, the community of movement ecologists is in a prime position to measure between-population movement responses and seek to understand the prevalence of movement-induced spatial distribution patterns reported here. Our hope is that the theoretical results presented here will serve as a motivating study for understanding the effect of between-population movement responses on spatial population dynamics in empirical systems, as well as highlighting the need for such studies if we are to understand accurately the drivers behind observed species distributions.

Acknowledgements JRP thanks the School of Mathematics and Statistics at the University of Sheffield for granting him study leave which has enabled the research presented here. MAL gratefully acknowledges the Canada Research Chairs program and Discovery grant from the Natural Sciences and Engineering Research Council of Canada.

Open Access This article is distributed under the terms of the Creative Commons Attribution 4.0 International License (http://creativecommons.org/licenses/by/4.0/), which permits unrestricted use, distribution, and reproduction in any medium, provided you give appropriate credit to the original author(s) and the source, provide a link to the Creative Commons license, and indicate if changes were made.

\section{References}

Adams ES (2001) Approaches to the study of territory size and shape. Ann Rev Ecol Syst 32:277-303

Albertsen CM, Whoriskey K, Yurkowski D, Nielsen A, Flemming JM (2015) Fast fitting of non-Gaussian state-space models to animal movement data via template model builder. Ecology 96(10):2598-2604

Alt W (1985) Degenerate diffusion equations with drift functionals modelling aggregation. Nonlinear Anal Theory Methods Appl 9(8):811-836

Araujo MB, Guisan A (2006) Five (or so) challenges for species distribution modelling. J Biogeogr 33(10):1677-1688

Avgar T, Baker JA, Brown GS, Hagens JS, Kittle AM, Mallon EE, McGreer MT, Mosser A, Newmaster SG, Patterson BR et al (2015) Space-use behaviour of woodland caribou based on a cognitive movement model. J Anim Ecol 84(4):1059-1070

Avgar T, Potts JR, Lewis MA, Boyce MS (2016) Integrated step selection analysis: bridging the gap between resource selection and animal movement. Methods Ecol Evol 7(5):619-630

Börger L, Dalziel BD, Fryxell JM (2008) Are there general mechanisms of animal home range behaviour? A review and prospects for future research. Ecol Lett 11(6):637-650. https://doi.org/10.1111/j.14610248.2008.01182.x 
Briscoe B, Lewis M, Parrish S (2002) Home range formation in wolves due to scent marking. Bull Math Biol 64(2):261-284. https://doi.org/10.1006/bulm.2001.0273

Burger M, Francesco MD, Fagioli S, Stevens A (2018) Sorting phenomena in a mathematical model for two mutually attracting/repelling species. SIAM J Math Anal 50(3):3210-3250

Durrett R, Levin S (1994) The importance of being discrete (and spatial). Theor Popul Biol 46(3):363-394

Evans TG, Diamond SE, Kelly MW (2015) Mechanistic species distribution modelling as a link between physiology and conservation. Conserv Physiol 3(1):cov056

Fagan WF, Lewis MA, Auger-Méthé M, Avgar T, Benhamou S, Breed G, LaDage L, Schlägel UE, Ww Tang, Papastamatiou YP, Forester J, Mueller T (2013) Spatial memory and animal movement. Ecol Lett 16(10):1316-1329. https://doi.org/10.1111/ele.12165

Fatima SH, Atif S, Rasheed SB, Zaidi F, Hussain E (2016) Species distribution modelling of Aedes aegypti in two dengue-endemic regions of pakistan. Trop Med Int Health 21(3):427-436

Fleming CH, Fagan WF, Mueller T, Olson KA, Leimgruber P, Calabrese JM (2015) Rigorous home range estimation with movement data: a new autocorrelated kernel density estimator. Ecology 96(5):11821188

Gallagher AJ, Creel S, Wilson RP, Cooke SJ (2017) Energy landscapes and the landscape of fear. Trends Ecol Evol 32(2):88-96

Gambino G, Lombardo MC, Sammartino M (2009) A velocity-diffusion method for a Lotka-Volterra system with nonlinear cross and self-diffusion. Appl Numer Math 59(5):1059-1074

Girardin L, Nadin G (2015) Travelling waves for diffusive and strongly competitive systems: relative motility and invasion speed. Eur J Appl Math 26(4):521-534

Giuggioli L, Potts JR, Rubenstein DI, Levin SA (2013) Stigmergy, collective actions, and animal social spacing. Proc Natl Acad Sci 110:16904-16909

Gotelli NJ, Stanton-Geddes J (2015) Climate change, genetic markers and species distribution modelling. J Biogeogr 42(9):1577-1585

Hastings A (1980) Disturbance, coexistence, history, and competition for space. Theor Popul Biol 18(3):363-373

Hastings A, Cuddington K, Davies KF, Dugaw CJ, Elmendorf S, Freestone A, Harrison S, Holland M, Lambrinos J, Malvadkar U et al (2005) The spatial spread of invasions: new developments in theory and evidence. Ecol Lett 8(1):91-101

Hays GC, Ferreira LC, Sequeira AM, Meekan MG, Duarte CM, Bailey H, Bailleul F, Bowen WD, Caley MJ, Costa DP et al (2016) Key questions in marine megafauna movement ecology. Trends Ecol Evol 31(6):463-475

Hooten MB, Johnson DS, McClintock BT, Morales JM (2017) Animal movement: statistical models for telemetry data. CRC Press, Boca Raton

Jiménez-Valverde A, Lobo JM, Hortal J (2008) Not as good as they seem: the importance of concepts in species distribution modelling. Divers Distrib 14(6):885-890

Kareiva P, Odell G (1987) Swarms of predators exhibit "prey taxis" if individual predators use area-restricted search. Am Nat 130(2):233-270

Kays R, Crofoot MC, Jetz W, Wikelski M (2015) Terrestrial animal tracking as an eye on life and planet. Science 348(6240):aaa2478

Kneitel JM, Chase JM (2004) Trade-offs in community ecology: linking spatial scales and species coexistence. Ecol Lett 7(1):69-80

Latombe G, Fortin D, Parrott L (2014) Spatio-temporal dynamics in the response of woodland caribou and moose to the passage of grey wolf. J Anim Ecol 83(1):185-198

Laundré JW, Hernández L, Ripple WJ (2010) The landscape of fear: ecological implications of being afraid. Open Ecol J 3:1-7

Lee J, Hillen T, Lewis M (2009) Pattern formation in prey-taxis systems. J Biol Dyn 3(6):551-573

Lewis M, Moorcroft P (2006) Mechanistic home range analysis. Princeton University Press, Princeton

Lewis MA, Murray JD (1993) Modelling territoriality and wolf-deer interactions. Nature 366:738-740

Lewis MA, Petrovskii SV, Potts JR (2016) The mathematics behind biological invasions, vol 44. Springer, Berlin

Lugo CA, McKane AJ (2008) Quasicycles in a spatial predator-prey model. Phys Rev E 78(5):051,911

Mainali KP, Warren DL, Dhileepan K, McConnachie A, Strathie L, Hassan G, Karki D, Shrestha BB, Parmesan C (2015) Projecting future expansion of invasive species: comparing and improving methodologies for species distribution modeling. Glob Change Biol 21(12):4464-4480 
Manly B, McDonald L, Thomas D, McDonald T, Erikson W (2002) Resource selection by animals: statistical design and analysis for field studies. Elsevier Academic Press, Chapman and Hall, New York

Michelot T, Langrock R, Patterson TA (2016) moveHMM: an R package for the statistical modelling of animal movement data using hidden markov models. Methods Ecol Evol 7(11):1308-1315

Mogilner A, Edelstein-Keshet L (1999) A non-local model for a swarm. J Math Biol 38(6):534-570

Murray JD (2003) Mathematical biology II: spatial models and biomedical applications. Springer, New York

Murrell DJ, Law R (2003) Heteromyopia and the spatial coexistence of similar competitors. Ecol Lett 6(1):48-59

Nathan R, Giuggioli L (2013) A milestone for movement ecology research. Move Ecol 1(1)

Nathan R, Getz WM, Revilla E, Holyoak M, Kadmon R, Saltz D, Smouse PE (2008) A movement ecology paradigm for unifying organismal movement research. Proc Natl Acad Sci 105(49):19052-19059. https://doi.org/10.1073/pnas.0800375105

Okubo A, Levin SA (2013) Diffusion and ecological problems: modern perspectives, vol 14. Springer, Berlin

Padrón V (1998) Sobolev regularization of a nonlinear ill-posed parabolic problem as a model for aggregating populations. Commun Partial Differ Equ 23(3-4):457-486

Padrón V (2004) Effect of aggregation on population recovery modeled by a forward-backward pseudoparabolic equation. Trans Am Math Soc 356(7):2739-2756

Painter KJ (2009) Continuous models for cell migration in tissues and applications to cell sorting via differential chemotaxis. Bull Math Biol 71(5):1117

Painter KJ, Hillen T (2011) Spatio-temporal chaos in a chemotaxis model. Physica D 240:363-375

Painter K, Bloomfield J, Sherratt J, Gerisch A (2015) A nonlocal model for contact attraction and repulsion in heterogeneous cell populations. Bull Math Biol 77(6):1132-1165

Pascual M (1993) Diffusion-induced chaos in a spatial predator-prey system. Proc R Soc Lond B 251(1330): $1-7$

Petrovskii SV, Morozov AY, Venturino E (2002) Allee effect makes possible patchy invasion in a predatorprey system. Ecol Lett 5(3):345-352

Potts JR, Lewis MA (2014) How do animal territories form and change? Lessons from 20 years of mechanistic modelling. Proc R Soc B 281(1784):20140,231

Potts JR, Lewis MA (2016a) How memory of direct animal interactions can lead to territorial pattern formation. J R Soc Interface 13:20160059

Potts JR, Lewis MA (2016b) Territorial pattern formation in the absence of an attractive potential. J Math Biol 72(1-2):25-46

Potts JR, Petrovskii SV (2017) Fortune favours the brave: movement responses shape demographic dynamics in strongly competing populations. J Theor Biol 420:190-199

Potts JR, Mokross K, Lewis MA (2014) A unifying framework for quantifying the nature of animal interactions. J R Soc Interface 11(96):20140,333

Potts JR, Börger L, Scantlebury DM, Bennett NC, Alagaili A, Wilson RP (2018) Finding turning-points in ultra-high-resolution animal movement data. Methods Ecol Evol 9(10):2091-2101

Rodríguez JP, Brotons L, Bustamante J, Seoane J (2007) The application of predictive modelling of species distribution to biodiversity conservation. Divers Distrib 13(3):243-251

Sherratt JA, Lewis MA, Fowler AC (1995) Ecological chaos in the wake of invasion. Proc Natl Acad Sci 92(7):2524-2528

Sherratt JA, Eagan BT, Lewis MA (1997) Oscillations and chaos behind predator-prey invasion: mathematical artifact or ecological reality? Philos Trans R Soc Lond Ser B Biol Sci 352(1349):21-38

Shi J, Xie Z, Little K (2011) Cross-diffusion induced instability and stability in reaction-diffusion systems. J Appl Anal Comput 1(1):95-119

Shigesada N, Kawasaki K, Teramoto E (1979) Spatial segregation of interacting species. J Theor Biol 79(1):83-99

Stewart IN (2015) Galois theory. CRC Press, Boca Raton

Sun GQ, Zhang J, Song LP, Jin Z, Li BL (2012) Pattern formation of a spatial predator-prey system. Appl Math Comput 218(22):11,151-11,162

Tania N, Vanderlei B, Heath JP, Edelstein-Keshet L (2012) Role of social interactions in dynamic patterns of resource patches and forager aggregation. Proc Natl Acad Sci 109(28):11,228-11,233

Theveneau E, Steventon B, Scarpa E, Garcia S, Trepat X, Streit A, Mayor R (2013) Chase-and-run between adjacent cell populations promotes directional collective migration. Nat Cell Biol 15(7):763 
Topaz CM, Bertozzi AL, Lewis MA (2006) A nonlocal continuum model for biological aggregation. Bull Math Biol 68(7):1601

Turing AM (1952) The chemical basis of morphogenesis. Phil Trans R Soc Lond B 237(641):37-72

Vanak A, Fortin D, Thakera M, Ogdene M, Owena C, Greatwood S, Slotow R (2013) Moving to stay in place-behavioral mechanisms for coexistence of African large carnivores. Ecology 94:2619-2631

White K, Lewis M, Murray J (1996) A model for wolf-pack territory formation and maintenance. J Theor Biol 178(1):29-43

Williams HJ, Taylor LA, Benhamou S, Bijleveld AI, Clay TA, de Grissac S, Demšar U, English HM, Franconi N, Gómez-Laich A, Griffiths RC, Kay WP, Morales JM, Potts JR, Rogerson KF, Rutz C, Spelt A, Trevail AM, Wilson RP, Börger L (in review) Optimising the use of bio-loggers for movement ecology research

Wilmers CC, Nickel B, Bryce CM, Smith JA, Wheat RE, Yovovich V (2015) The golden age of bio-logging: how animal-borne sensors are advancing the frontiers of ecology. Ecology 96(7):1741-1753

Publisher's Note Springer Nature remains neutral with regard to jurisdictional claims in published maps and institutional affiliations. 